\title{
ARTICLE Role of gamma-amino-butyric acid in the dorsal anterior cingulate in age-associated changes in cognition
}

\author{
Stefano Marenco iD $^{1}$, Christian Meyer ${ }^{1}$, Jan Willem van der Veen ${ }^{2}$, Yan Zhang ${ }^{2}$, Ryan Kelly ${ }^{1}$, Jun Shen ${ }^{2}$, Daniel R. Weinberger ${ }^{3,4,5,6}$, \\ Dwight Dickinson ${ }^{1}$ and Karen F. Berman ${ }^{1}$
}

\begin{abstract}
GABAergic mechanisms have been shown to contribute to cognitive aging in animal models, but there is currently limited in vivo evidence to support this relationship in humans. It is also unclear whether aging is associated with changes in GABA levels measured with proton magnetic resonance spectroscopy (MRS). Spectral-editing MRS at $3 \mathrm{~T}$ was used to measure GABA in the dorsal anterior cingulate cortex (dACC) for a large sample of healthy volunteers $(N=229)$ aged $18-55$. In a subset of 171 participants, age effects on several cognitive tasks were studied. We formally tested whether the MRS measures mediated the relationship between age and cognition. Robust associations of age with performance were found for the Wisconsin Card Sorting Test ([WCST], $p<0.0001$ ). Age was also significantly associated with declining levels of GABA in the dACC $(p<0.001)$, and GABA levels significantly predicted WCST performance $(p<0.0004)$. Mediation analysis revealed that GABA in the dACC mediated the effect of age on WCST performance $(p<0.01)$. Other metabolites were similarly associated with age, but only GABA and creatine levels were significantly associated with WCST performance. No association with age or cognitive performance was found in a frontal white matter control region in a subset of participants. The association of GABA with WCST performance was not related to the amount of brain atrophy associated with aging as measured by the proportion of CSF, gray, and white matter in the MRS voxel. These results implicate GABAergic and possibly energetic metabolism in the dACC as mechanisms of age effects in executive function.
\end{abstract}

Neuropsychopharmacology (2018) 43:2285-2291; https://doi.org/10.1038/s41386-018-0134-5

\section{INTRODUCTION}

Cognitive aging is likely a multifactorial phenomenon with complex biochemical-structural underpinnings. Defining the mechanisms that contribute to cognitive aging is critical in order to find targets for treatment and prevention of cognitive disorders that are age-associated, but may also offer insights into major mental illness that may be impacted by accelerated aging [1] and into the pathogenesis of dementia [2]. Alterations in inhibitory mechanisms and cortical excitability are thought to play a role in cognitive aging, but this is based primarily on evidence from animal models and poorly translated to human. Critically, the basic mechanisms of this alteration remain unknown, with partly contradictory findings. For example, a rich body of work has shown that, in rodent models, altered spatial memory occurring with age is accompanied by a reduction in the number of interneurons expressing the gamma-amino-butyric acid (GABA) synthetic enzyme glutamic acid decarboxylase (GAD) in the medial temporal lobe [3], and by a host of neurophysiological changes indicative of reduced GABAergic transmission both at GABA-a and GABA-b receptors [4]. Studies in visual cortex of cats [5] and monkeys [6] are also consistent with reduced GABAergic neuron number and function, respectively.
These results in medial temporal lobe and occipital cortex contrast with findings in the prefrontal cortex, where aging in rats is associated with increased GAD protein expression [7], reduced GABA transporter, and increased GABA-b receptors [8].

In post-mortem human brain, reductions of GABA-related mRNA transcripts with advancing age have been reported [912] in microarray studies, but these findings have not been supported by the existing RNA-sequencing (RNAseq) studies [1315], with the exception of Dillman et al. [16]. This discrepancy may depend on the much higher statistical bar for differential expression in RNAseq studies as compared to microrrays (due to the increased number of transcripts explored), the lack of a specific hypothesis limited to GABA-related transcripts or the derivation of a subset of age-sensitive transcripts from other tissues where genes expressed primarily in neurons may be underrepresented $[13,14]$.

There is generally little in vivo data to support or refute the available animal and post-mortem data. GABA levels measured with magnetic resonance spectroscopy (MRS) in vivo [17] may be useful in supporting or refuting the role of GABA in aging. Current evidence derived from measurements conducted in the dorsal anterior cingulate cortex (dACC) supports a decline of GABA levels

\footnotetext{
${ }^{1}$ Clinical and Translational Neuroscience Branch, NIMH-Intramural Research Program (IRP), Bethesda, MD 20892, USA; ${ }^{2}$ Magnetic Resonance Spectroscopy Core, NIMH-IRP, Bethesda, MD 20892, USA; ${ }^{3}$ Lieber Institute for Brain Development, Johns Hopkins Medical Campus, Baltimore, MD 21205, USA; ${ }^{4}$ Departments of Psychiatry and Behavioral

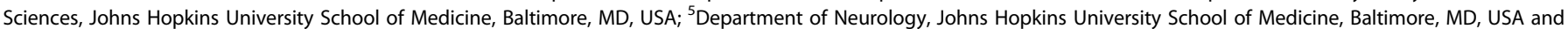
${ }^{6}$ Department of Neuroscience, Johns Hopkins University School of Medicine, Baltimore, MD, USA Correspondence: Stefano Marenco (marencos@mail.nih.gov)
}

Received: 29 January 2018 Revised: 13 May 2018 Accepted: 18 June 2018 Published online: 3 July 2018 


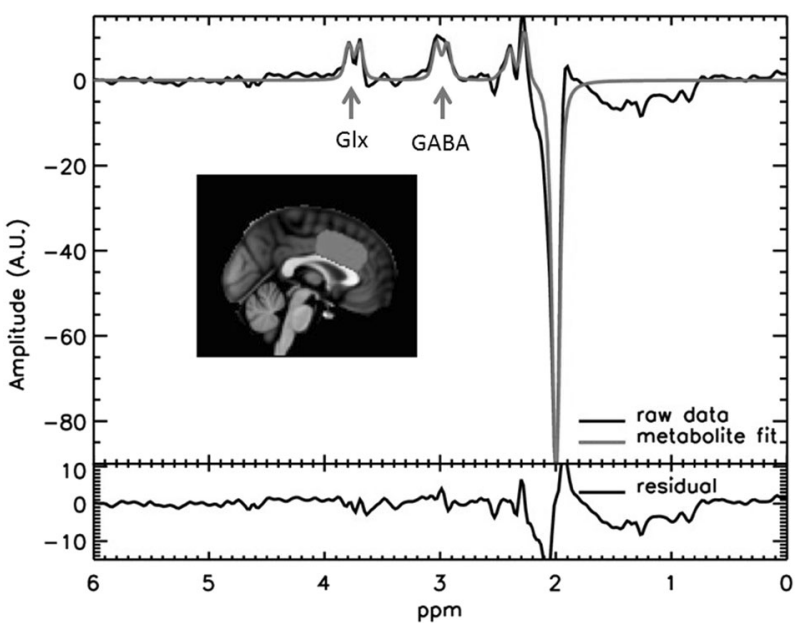

Fig. 1 Location of the gray matter voxel (red) and representative "difference" spectrum. The voxel shown here is the average location of all individuals after registration to $\mathrm{MNI}$ space [see ref. [28] for details]

with age in vivo [18-20], although others [21-24] found no change, and one study found an increase [25]. Porges et al. [19] also assessed the functional consequences of reduced GABA levels with age, showing that cognitive decline in the 50-90 age range is associated with reduced GABA levels.

We hypothesized not only that GABA levels in the prefrontal cortex would be associated with cognitive abilities and age, even before age 55 , but also that they would statistically mediate the relationship of age with cognitive function. We also hypothesized that this effect would be independent of brain atrophy and would be specific to GABA as compared to other metabolites measured in the same region. We also predicted that the same effect would not be found in a control region (the adjacent white matter (WM)), where GABA levels should not be critical to tissue function. We tested these hypotheses in the largest sample of healthy volunteers to date $(N=229)$ acquired with in vivo GABA MRS. Of these participants, at least 141 also received a comprehensive cognitive assessment yielding six cognitive dimension composite scores and a measure of general cognitive ability.

\section{METHODS}

\section{Participants}

The 229 healthy volunteers (mean age $=30 \pm 9.2$ [standard deviation; SD] years, range 18-54 years; 127 females) participated in Institutional Review Board-approved protocols 95-M-0150 (NCT00001486) and 00-M-0085 (NCT00004571) between February 2008 and May 2015. Subsets of this data collection were used for previous reports on genetic association of GABA levels $[26,27]$, or on GABA abnormalities in schizophrenia [ $N=183$ controls [28]]. All gave written informed consent and had MRS data that survived our stringent quality control [28] (Supplementary Material). Exclusion criteria were as follows: current or prior psychiatric history (assessed by a Structured Clinical Interview for DSM Disorders); substance abuse; use of psychotropic medication at the time of the scan; contraindications to magnetic resonance imaging (MRI); history of head trauma; abnormalities of the brain on MRI; Wechsler Adult Intelligence Scale intelligence quotient (IQ) > 70 (WAIS IQ) or learning disability history; positive toxicology for common drugs of abuse; and medical conditions affecting brain perfusion and function.

\section{Cognitive variables}

Participants completed a comprehensive battery of cognitive tests [29]. Here we examined composite scores derived from this battery for six cognitive dimensions-verbal memory, working memory performance on the $N$-back task, visual memory, processing speed, performance on the Wisconsin Card Sorting Test ([WCST], a measure of executive function), and digit span. We also examined a four-subtest estimate of WAIS IQ [30] as a proxy for broad cognitive ability.

\section{MRS variables}

MRS acquisition. Metabolite concentrations were collected with single-voxel MRS on a 3 T GE scanner using a quadrature transmitreceive head coil. To position the spectroscopic voxels, high resolution T1 structural images were acquired using a threedimensional spoiled gradient (SPGR) pulse sequence ( $T R=24 \mathrm{~ms}$, $\mathrm{TE}=3.2 \mathrm{~ms}$, flip angle $=17^{\circ}$, in-plane resolution $=0.9 \times 0.9 \mathrm{~mm}^{2}$, $192 \times 256$ matrix, field of view $240 \mathrm{~mm}$, and slice thickness $2 \mathrm{~mm}$ ). MRS spectra were acquired from two voxels, each measuring 2 $\mathrm{cm} \times 2 \mathrm{~cm} \times 4.5 \mathrm{~cm} \quad\left(18 \mathrm{~cm}^{3}\right)$. One voxel included mainly gray matter (GM) of the anterior cingulate and medial prefrontal cortex. The structural image that served to localize the voxel was first tilted in order to be parallel to the anterior portion of the corpus callosum. The GM voxel was then placed such that it symmetrically straddled the midline, with its anterior edge aligned to the genu of the corpus callosum, and its lower bound just above the corpus callosum (Fig. 1). In a subset of participants $(N=113)$, a second voxel was placed adjacent to the first, in the right frontal WM (Supplementary Material and Fig.S1).

An interleaved PRESS-based J-editing method was used [31]. Each voxel was scanned 784 times $(384 \times 2$ interleaved +16 water reference scans) with a TE of $68 \mathrm{~ms}$ and a TR of $1.5 \mathrm{~s}$. In all, 384 water-suppressed acquisitions were collected with the "editing pulse" on (1.89 ppm), another 384 with the editing pulse off (20.3 ppm), and 16 with no water suppression.

MRS data processing. Preprocessing of MRS data was described in Geramita et al. [31] and is detailed here in Supplementary Materials. After spectral fitting, using the averaged "editing-pulseoff" signal, model functions for the choline (Cho), creatine, and $\mathrm{N}$ acetyl-aspartate (NAA) peaks were fitted using fixed relative frequency separations based on literature values and a shared lineshape. Similar assumptions were applied to determine the frequency positions of GABA+ and Glx in the "difference" spectrum. The processing yielded arbitrary, unscaled values for GABA+, partially co-edited Glx, Cre, NAA, and Cho. The GABA peak in the difference spectrum contains $40 \%$ macromolecules [32], and will therefore be referred to as GABA+.

Metabolites derived from the J-edited sequence were referenced to Cre as well as to water. Our primary measure of interest was the ratio of GABA to Cre. Determination of the water signal is described in the Supplementary information.

Determination of voxel tissue composition. We segmented the SPGR structural scans using SPM5 [33]. In the output image, each voxel was given a grayscale intensity that labeled it as cerebrospinal fluid (CSF), GM, or WM. The percent tissue makeup of the spectroscopy voxel was extracted using software written in-house in IDL. The percentage of GM in the tissue (GM/ tissue) was calculated by dividing GM by the sum of GM and WM.

\section{Statistical analysis}

Cognitive variables. Because our main focus was on investigating how age-related changes of $\mathrm{GABA}+$ might affect cognitive decline, we first determined which cognitive variables were robustly associated with age in the current sample. Backward stepwise multiple regression models using age, sex, and years of education as independent variables were run for each of the seven cognitive variables, allowing for all two-way interactions, removing effects when $p>0.1$ and forcing the main effects of 
Role of gamma-amino-butyric acid in the dorsal anterior cingulate in... $\mathrm{S}$ Marenco et al.

independent variables in the model. All two-way interactions that were highly collinear with one of the main effects (tolerance value $<0.1$ or variance inflation factor $>5$ ) were removed from the model to improve interpretability of the data. If the overall model and the effect of age were significant beyond a conservative multiple comparison correction $(p<0.007$ because we regressed age on seven cognitive scores), the cognitive variable was retained for further analysis of the effects of GABA+.

Relationship of GABA to age and cognition. Outliers (defined as values $>3$ SDs from the mean) from all variables of interest were excluded prior to any analysis. The effects of age, sex, and \%GM/ tissue on $\mathrm{GABA}+/$ Cre were explored with a backward stepwise multiple regression model, similar to the models described above for the cognitive variables. The same statistical strategy was used to assess the relationship of GABA+/Cre to cognitive performance, using age and sex as covariates.

\section{Tests of specificity}

Specificity of GABA+/Cre vs. other metabolites: To test the specificity of the relationship of age to GABA+/Cre as compared to other ratios, similar multiple regression models as described above were run for each metabolite ratio (NAA/Cre, Cho/Cre, and $\mathrm{Glx} / \mathrm{Cre}$ ), and the $95 \%$ confidence intervals ( $\mathrm{Cls}$ ) of the normalized regression coefficients (beta) for age were compared to each other. Absence of overlap in the $95 \% \mathrm{Cls}$ was considered as evidence of specificity. To test the specificity of the relationship of $\mathrm{GABA}+/ \mathrm{Cre}$ to cognition, all the other metabolite ratios were added to the model predicting WCST performance, using backward stepwise multiple regression, as above.

Specificity of ratios to creatine vs. ratios to water: Similar statistics as mentioned above for ratios to Cre were calculated for ratios of all metabolites to water. Ratios to water provide an important confirmation of the directionality of findings for ratios to Cre. Similar directionality of both ratios to Cre and water is usually supportive of the numerator being mainly responsible for the finding, while opposite directionalities of the metabolite ratio to Cre and Cre/water support an effect of creatine.

Specificity of region of interest: Regional specificity was tested by applying the same analytic procedure as above for GABA+/Cre to the subset of individuals with WM measures.

Specificity with regards to overall tissue changes: Because sulcal widening occurs with age has been associated with declining cognitive performance [34], and our voxel straddled the midline, we tested for the association of CSF, GM, and WM content of the measurement voxel with age and cognition (again with the same backward stepwise regression already described).

Mediation analysis. Mediation analysis provides an approach for testing whether the effects of an independent variable on a dependent outcome of interest are transmitted through intervening variables or "mediators" [35]. Here GABA+/Cre was further investigated as a possible mediator of the age-cognitive associations that survived multiple comparison corrections $(p<0.007)$ via mediation analysis [35]. The dependent variable was the cognitive variable of interest, and the independent variables were age and GABA+/Cre. We tested for the significance of the indirect mediation effect using PROCESS for SPSS [36]; version 2.16, released July 2016 (http://processmacro.org/download.html). Further information on this statistical procedure is in Supplementary Material.

PROCESS uses an ordinary least squares-based path-analytic framework for estimating direct and indirect effects in mediator models. Bootstrapping (resampling) is used to generate nonparametric $\mathrm{Cls}$ for inference about indirect effects, including
Table 1. Demographic and cognitive characteristics, and MRS values

\begin{tabular}{llll}
\hline & $N$ & Mean & SD \\
\hline Male/female & $102 / 127$ & & \\
Age & 229 & 30 & 9.2 \\
Years of education & 176 & 16.3 & 2.4 \\
WAIS IQ & 175 & 106.9 & 10.6 \\
Verbal memory factor & 174 & 25.3 & 7.6 \\
$N$-back factor & 170 & 25.7 & 8.7 \\
Visual memory factor & 141 & 294.1 & 120.6 \\
Processing speed factor & 176 & 3.9 & 0.8 \\
Card sorting factor & 171 & 147.3 & 55.8 \\
\%CSF & 225 & 12 & 0.035 \\
\%GM/tissue & 229 & 70 & 0.052 \\
GABA+/Cre & 228 & 0.096 & 0.0088 \\
\hline
\end{tabular}

Years of education and WAIS IQ are reported to provide a general description of this sample. WAIS IQ was estimated based on four subtests of the Wechsler Adult Intelligence Scale. $N=$ number of subjects for which the information was available or who were included in the study after outlier removal. There were four outliers identified for \%CSF and one for $\mathrm{GABA} / \mathrm{Cre}$. Means and SD of the factors do not conform to an interpretable scale because of various transformations applied to the data to achieve normal distribution.

CSF cerebrospinal fluid content in the MRS voxel (four statistical outliers rejected), GM/tissue proportion of gray matter in the MRS voxel, Cre creatine

various measures of effect size. Using PROCESS, we generated $99 \% \mathrm{Cls}$ for the indirect effect based on 5000 bootstrap samples.

\section{RESULTS}

Subject descriptors and MRS values for the sample are shown in Table 1.

Association of cognitive variables with age

The number of subjects with cognitive data varied between 141 for the visual memory and 176 for the processing speed scores. The subsample with available cognitive data did not differ from the rest of the sample in terms of age $(p=0.72)$, years of education $(p=0.22)$, sex $(p=0.28)$, or race $(p=0.07)$. In this sample, the standardized composite score derived from WCST performance (including correct trials, categories achieved, and perseverative errors) was the only cognitive variable that was significantly associated with age after correction for multiple comparisons $\left(N=171\right.$, adjusted $R^{2}=0.074$, overall $p<0.002$, univariate $p$ for age $<0.0002$ ). IQ and $N$-back scores were nominally associated with age $[p=0.03]$, but the result did not survive correction for multiple comparisons. All other $p$ values for the univariate effect of age were $>0.41$. The statistical significance of the relationship between WCST score and age persisted even when only individuals who had complete scores on all cognitive variables $(N=134$, effect of age $p<0.003)$ were considered, and WCST performance remained the only significant variable in this subset analysis.

Association of $\mathrm{GABA}+/$ Cre with age and cognition GABA+/Cre was significantly associated with age $(\beta=-0.29, p=$ 0.00001 , with sex and \%GM/tissue included in the model, Table 2 ) and with WCST performance $(\beta=0.19, p=0.01$, age and sex included in the model, Table 3 ).

Tests of specificity

Table 2 shows the relationship to age of all metabolite ratios. All ratios to Cre with the exception of Cho/Cre were significantly 


\begin{tabular}{|c|c|c|c|c|c|c|c|}
\hline \multirow{8}{*}{ 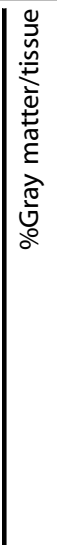 } & \multirow{8}{*}{ 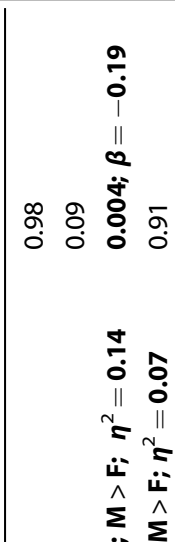 } & \multirow{8}{*}{ 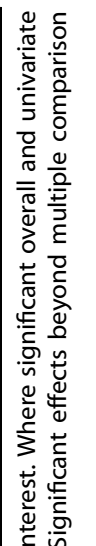 } & \multicolumn{5}{|c|}{$\begin{array}{l}\text { Table 3. Relationship of metabolites ratios to Cre with WCST } \\
\text { performance }\end{array}$} \\
\hline & & & & $p$ & Beta & \multicolumn{2}{|c|}{$\begin{array}{l}95 \% \text { confidence } \\
\text { intervals of beta }\end{array}$} \\
\hline & & & Sex & 0.43 & -0.064 & -0.223 & 0.096 \\
\hline & & & Age & 0.007 & -0.216 & -0.373 & -0.059 \\
\hline & & & NAA/cre & 0.34 & 0.078 & -0.082 & 0.238 \\
\hline & & & Cho/Cre & 0.84 & -0.017 & -0.179 & 0.145 \\
\hline & & & Glx/Cre & 0.93 & -0.008 & -0.181 & 0.165 \\
\hline & & & $\begin{array}{l}\text { For the over } \\
\text { way interacti }\end{array}$ & sig & Signific & ings in bo & wo- \\
\hline
\end{tabular}

negatively associated with age and accounted for between 8 and $11 \%$ of the variance in age. Table 3 summarizes the association of metabolite ratios to WCST performance (dependent variable). When all the ratios were entered in the model, GABA+/Cre was the only metabolite to have a statistically significant association with WCST performance. The $95 \% \mathrm{Cls}$ of the standardized regression coefficient overlapped across metabolite ratios, indicating limited specificity of $\mathrm{GABA}+/ \mathrm{Cre}$ vs. the other metabolite ratios.

Tables S1 and S2 illustrate the association of metabolite/water ratios with age and WCST performance, respectively. GABA + /water was related to age with the same directionality as GABA+/Cre; however, this association was less significant than GABA+/Cre $(\beta=-0.15, p=0.02)$. Cre/water was positively associated with age $(\beta=0.13, p=0.04$, Table S1). GABA+/water and Cre/water were significantly associated with WCST performance independently of each other, since their $95 \% \mathrm{Cls}$ did not overlap (Table S2). The Cls of the other metabolites referenced to water, however, did overlap with both GABA+/water and Cre/water, again indicating limited specificity.

In the WM subsample (demographics in Table S3), GABA+/Cre was associated with age $(\beta=-0.24, p=0.009)$ but not with WCST performance $(\beta=-0.12, p=0.25)$. The latter does not support a role for WM GABA+/Cre in decline of WCST performance related to aging.

The proportions of CSF and GM in the MRS voxel were oppositely associated with age (Table S4), but were not associated with WCST performance ( $p>0.78$, Table S5), indicating that tissue composition alone could not explain significant portions of the variance in WCST performance beyond that explained by age alone. A model with WCST as the dependent variable and sex, age, $\mathrm{GABA}+/ \mathrm{Cre}, \mathrm{CSF}$, and GM fractions as independent variables, was significant (adjusted $R^{2}=0.12, F_{(5,162)}=5.4$, overall $p=0.0001$ ), and GABA $+/$ Cre was the only variable to be statistically significant above and beyond the other independent variables $(\beta=0.22$, partial $\eta^{2}=0.047, p=0.005$ ). The tissue fractions all had $p>0.06$. This is evidence for an association of GABA+/Cre with WCST performance, independent of overall tissue changes.

Mediation analysis

GABA+/Cre was associated both with age and with cognitive performance on the WCST. We therefore used formal mediation analysis to test whether GABA+/Cre mediated the effect of age on the WCST factor. The model including both age and GABA+/Cre was significant $(F[2,168]=9.42, p=0.0001)$, accounting for approximately $10 \%$ of the variance in WCST performance $\left(R^{2}=\right.$ $0.101)$. The direct effect of age was reduced by the inclusion of $\mathrm{GABA}+/$ Cre in this model, but remained significant $(t[170]=2.30$, $p=0.02)$. The indirect effect of age mediated through GABA $+/$ Cre on WCST was significant $p<0.01$ (i.e., the $99 \%$ Cls did not overlap 


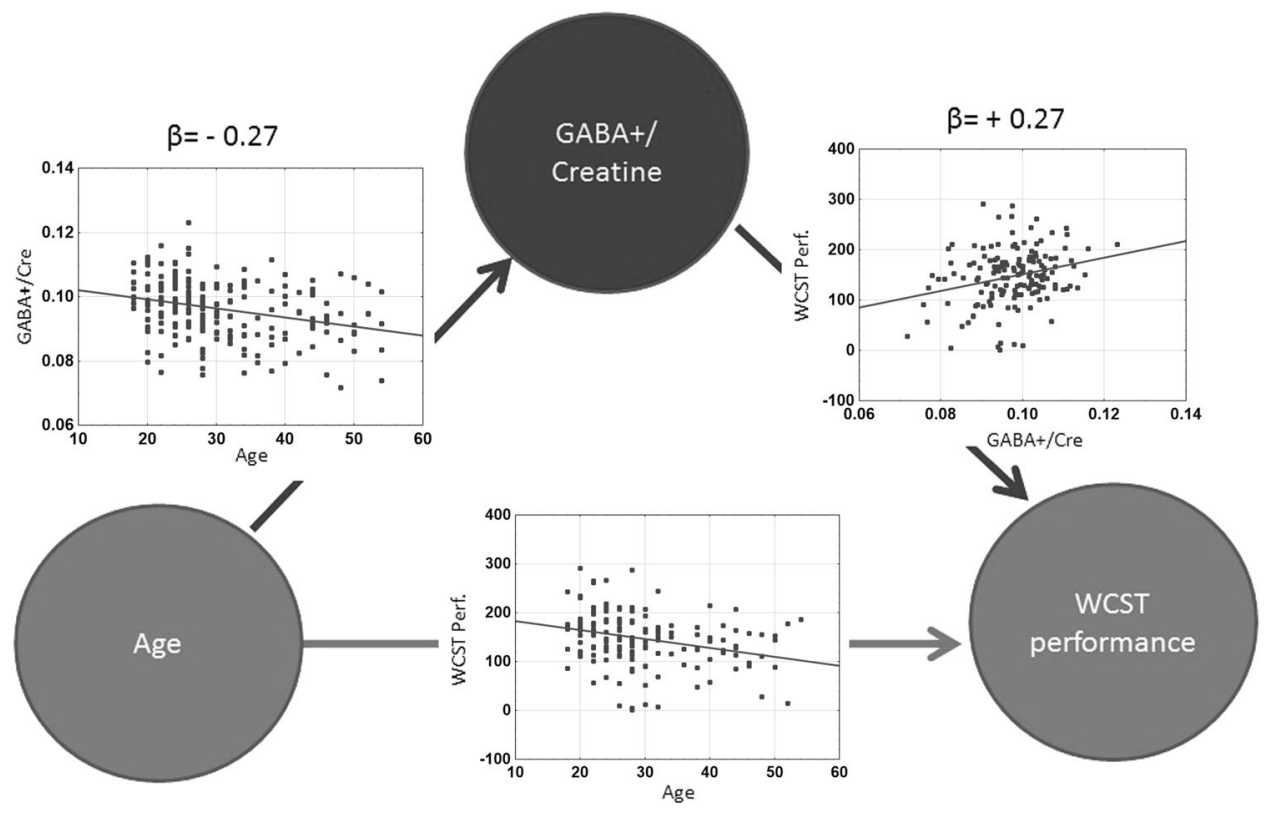

$\beta=-0.24-->-0.18$ (after inclusion of GABA+/Cre)

Fig. 2 Relationship between age and WCST performance, and mediation effects of GABA+/Cre. Raw values are shown in the plots. Beta values are from regression analyses. The $\beta$ value of the age-WCST performance regression was -0.24 when only age was included as an independent variable in the model, and -0.18 when $\mathrm{GABA}+/$ Cre was added to the regression model

zero: effect $=-0.0061, \mathrm{SE}=0.0027,99 \% \mathrm{Cl}:-0.016$ to -0.001 ). The estimated $R^{2}$ for the indirect effect of GABA+/Cre was 0.029 (SE $=0.016,99 \% \mathrm{Cl}: 0.004$ to 0.094$)$. These results indicate that a significant mediation effect occurred. Figure 2 summarizes these results.

\section{DISCUSSION}

In 229 healthy volunteers aged 18-55 (mean $30+9.2$ ), we found that measures of GABA in the dACC decrease with age. In a subset of 171 subjects, age was associated with reduced performance on the WCST. The WCST is a problem-solving task, thought to index executive function, in which the subject must adapt dynamically to ongoing feedback in order to correctly assign cards to categories (color, shape, or number) that shift unpredictably during task performance. This task has been found to be sensitive to damage to the frontal lobe and has been associated with brain activation in multiple cortical areas, including the ACC [37]. GABA/ Cre in the dACC was also associated with WCST performance and statistically mediated the effects of age on WCST performance.

Association of cognitive variables with age The existence of age effects on WCST performance is well established [38]. Other cognitive variables, including processing speed and verbal memory, were not significantly associated with age in our study cohort. Several factors in addition to stringent multiple comparisons corrections likely account for this. First, the sample had a restricted age range (18-54 years, with two-thirds of the sample between 20 and 39), and it may be that the WCST is more sensitive than other cognitive measures to age-related performance changes within this age range [39]. Also, we excluded individuals with $\mathrm{IQ}<70$, evidence of learning disabilities, and/or age-related brain abnormalities, such as WM hyperintensities, all of which might account for some age-related cognitive decline in an unselected sample.

Effects of age on metabolites

Both our measures of GABA+ decreased with advancing age. This is consistent with findings by Gao et al. [18] for GABA+/Cre, and those of Rowland et al. [20] for GABA/water, but not with those of others $[22,24,25]$ for GABA/water. The most significant difference between our study and the ones mentioned above may be in the age-based correction of the T2 of water that we applied. We show here (see Supplementary Material) that the T2 of water declines significantly with age in GM; this observation has important implications for all MRS signals reported as ratios to water (sometimes referred to as "absolute" values) since this approach would result in inflated estimates of metabolite concentrations at older ages. Had we not performed the correction for age-related decline in $\mathrm{T} 2$ of water, GABA+/water would not have been associated with age in a statistically significant manner (results not shown). It is important to note that the T2 of water has been reported to increase with age using imaging methods such as turbo spin echo or gradient recalled sequences $[40,41]$. We used the same spectroscopic PRESS sequence for the water T2 calculation and for the metabolite determination. Spectroscopic sequences may be weighted toward $\mathrm{T}^{*}$, which has been shown to decrease with age $[40,42]$, and may therefore be a more suitable method for correction of T2 of water in MRS studies.

\section{Associations of GABA with cognition}

Here we observed a statistically significant association of GABA $+/$ Cre with WCST performance, accounting for about $6.2 \%$ of the variance, which survived covariation for sex and age. This is consistent with the relationship between GABA+ and a more general cognitive task (the Montreal Cognitive Asessment) found by Porges et al. [19] in a group of older patients. The fact that two well-powered studies, ours and that of Porges et al. [19], converge in finding a relationship between anterior cingulate GABA and a complex cognitive ability declining with age adds import to this result, especially given the frequent lack of replicability of imaging findings. Moreover, the overlap between the findings of these two studies bolsters the argument that GABAergic transmission is involved in cognitive decline well before the appearance of frank cognitive deficits. It is therefore possible that alterations of GABA transmission might be very early occurrences in the chain of events leading to cognitive disturbances of aging. 
No other ratio to Cre reached statistical significance, although $95 \% \mathrm{Cls}$ for betas overlapped across ratios. The latter finding may be due to (1) the rather shallow slope of the association of GABA +/Cre with WCST performance; (2) to the fact that all ratios have the same denominator, which reduces their mathematical independence; and (3) to the fact that there is a biological correlation among some of the metabolites (e.g., NAA has been associated with glutamate levels [43] and GABA and Glu are in the same metabolic pathway).

The WCST is a widely used index of executive function and dynamic, feedback-guided problem solving. The association of GABA+/Cre with WCST performance in the dACC was not found in the WM, likely implying a selective cortical mechanism consistent with the higher concentrations of GABA and Cre in the cortex as compared to WM [31]. The association of GABA+/Cre with WCST performance was likely due to both the numerator and the denominator of the ratio, since both GABA+/water and Cre/water were significant and fully independent of each other in the results shown in Table S2 (they explained about $2.5 \%$ and $4 \%$ of the variance in WCST performance, respectively, while age explained about 5\%).

Proportion of CSF and GM in the voxel were associated with age, as expected, but not with WCST performance when age was accounted for. Thus, we lacked statistical evidence to support adding CSF or GM proportion in our models. However, we cannot rule out that with improved measurement precision, effects of CSF and GM might have been detectable. These variables did not account for a significant portion of variance in WCST performance when introduced in the same model as GABA+/Cre, therefore their potential role as independent mediators of the relationship of age to WCST performance is likely to be weaker than, and independent of, GABA+/Cre.

\section{Mediation analysis}

GABA+/Cre in the dACC was a statistically significant mediator of the association between age and WCST performance (Table 4), suggesting that reduced GABA and increased Cre in the dACC are factors contributing to poorer problem solving and cognitive flexibility with age. Few other investigations have explored mediation effects of metabolites measured with MRS on cognitive function. Gomar et al. [44] examined the precuneus in a sample older than 50 years of age and found that associations of Cho/Cre with a composite measure of broad cognitive ability varied as a function of APOE genotype (including APOE-4), but these investigators did not examine GABA. CSF and measures of Alzheimer pathology (e.g., estimates of amyloid concentration) were not included in this analysis. We found only weak evidence for a decline in IQ with age $(p<0.01$ uncorrected) within the relatively younger age range considered here. Rowland et al. [20] demonstrated a modest role for GABA in the dACC in predicting verbal working memory performance, but these authors did not test for age effects. Porges et al. [19] did not report beta values making it difficult to determine if their results were likely to support a mediation effect similar to the one found here.

Our findings are in line with literature from animal models showing a contribution of GABA-related deficits of inhibition to cognitive aging [45]. Since spectroscopy detects total metabolite levels rather than synaptically active GABA, our data cannot be interpreted to reflect inhibitory function per se. Despite this consideration, however, experiments in animal models and cell culture where intracellular concentration of GABA was increased by inhibition of GABA transaminase resulted in increased cytosolic and vescicular GABA [46], as well as depolarization- and electrical stimulation-evoked release of endogenous GABA [47, 48], indicating some degree of equilibrium between intra- and extracellular compartments. The MRS technique used here does not provide information on GABA-a or GABA-b receptors, which are important in modulating cognition, at least in animal models [49]. Our findings implicating GABAergic transmission might also be compatible with the observation that $\beta$-amyloid accumulation can cause selective dysfunction of parvalbumin positive inhibitory interneurons [2], and that inducing overexpression of sodium channels (Nav1.1, produced by SCN1A) in these cells can ameliorate memory dysfunction in animal models of Alzheimer disease [50,51]. A contribution of energy-related mechanisms, possibly indirectly indexed by the Cre peak, is also suspected to play a part in cognitive aging $[52,53]$, and a hypothesis has been advanced that inhibitory interneurons with high energy demands are particularly important for cognitive decline [54].

Some limitations of this study are discussed in the Supplementary Material.

\section{CONCLUSIONS}

In summary, cognitive decline with age through middle adulthood, as assessed by WCST performance, appears to be partly mediated by GABA+/Cre levels, pointing to specific biologic mechanisms in the dACC. Further dissection of these mechanisms in animal models could be important in understanding the biology of disease associated with aging. Moreover, longitudinal studies will play a central role in confirming these findings.

\section{ACKNOWLEDGEMENTS}

We thank Ena Xiao, Susie Kuo and Brad Zoltick for help with database development and maintenance. This research was entirely supported by the Intramural Research Program of the NIMH (protocols 95-M-0150 [NCT00001486], 00-M-0085 [NCT00004571], ZIA MH002942-05 CTNB).

\section{ADDITIONAL INFORMATION}

Supplementary Information accompanies this paper at (https://doi.org/10.1038/ s41386-018-0134-5).

Competing interests: The authors declare no competing interests.

Publisher's note: Springer Nature remains neutral with regard to jurisdictional claims in published maps and institutional affiliations.

\section{REFERENCES}

1. McKinney $B C$, Oh $\mathrm{H}$, Sibille E. Age-by-disease biological interactions: implications for late-life depression. Front Genet. 2012;3:237.

2. Palop JJ, Mucke L. Network abnormalities and interneuron dysfunction in Alzheimer disease. Nat Rev Neurosci. 2016;17:777-92.

3. Spiegel AM, Koh MT, Vogt NM, Rapp PR, Gallagher M. Hilar interneuron vulnerability distinguishes aged rats with memory impairment. J Comp Neurol. 2013;521:3508-23.

4. McQuail JA, Frazier CJ, Bizon JL. Molecular aspects of age-related cognitive decline: the role of GABA signaling. Trends Mol Med. 2015;21:450-60.

5. Hua T, Kao C, Sun Q, Li X, Zhou Y. Decreased proportion of GABA neurons accompanies age-related degradation of neuronal function in cat striate cortex. Brain Res Bull. 2008;75:119-25.

6. Leventhal AG, Wang Y, Pu M, Zhou Y, Ma Y. GABA and its agonists improved visual cortical function in senescent monkeys. Science. 2003;300:812-5.

7. Banuelos C, Beas BS, McQuail JA, Gilbert RJ, Frazier CJ, Setlow B, et al. Prefrontal cortical GABAergic dysfunction contributes to age-related working memory impairment. J Neurosci. 2014;34:3457-66.

8. McQuail JA, Banuelos C, LaSarge CL, Nicolle MM, Bizon JL. GABA(B) receptor GTPbinding is decreased in the prefrontal cortex but not the hippocampus of aged rats. Neurobiol Aging. 2012;33:1124 e1121-1112.

9. Erraji-Benchekroun L, Underwood MD, Arango V, Galfalvy H, Pavlidis P, Smyrniotopoulos $\mathrm{P}$, et al. Molecular aging in human prefrontal cortex is selective and continuous throughout adult life. Biol Psychiatry. 2005;57:549-58.

10. Loerch PM, Lu T, Dakin KA, Vann JM, Isaacs A, Geula C et al. Evolution of the aging brain transcriptome and synaptic regulation. PLoS ONE. 2008;3:e3329.

11. Lu T, Pan Y, Kao SY, Li C, Kohane I, Chan J, et al. Gene regulation and DNA damage in the ageing human brain. Nature. 2004;429:883-91. 
12. Berchtold NC, Cribbs DH, Coleman PD, Rogers J, Head E, Kim R, et al. Gene expression changes in the course of normal brain aging are sexually dimorphic. Proc Natl Acad Sci USA. 2008;105:15605-10.

13. Glass D, Vinuela A, Davies MN, Ramasamy A, Parts L, Knowles D, et al. Gene expression changes with age in skin, adipose tissue, blood and brain. Genome Biol. 2013;14:R75.

14. Sood S, Gallagher IJ, Lunnon K, Rullman E, Keohane A, Crossland H, et al. A novel multi-tissue RNA diagnostic of healthy ageing relates to cognitive health status. Genome Biol. 2015;16:185.

15. Brinkmeyer-Langford CL, Guan J, Ji G, Cai JJ. Aging shapes the population-mean and -dispersion of gene expression in human brains. Front Aging Neurosci. 2016;8:183.

16. Dillman AA, Majounie E, Ding J, Gibbs JR, Hernandez D, Arepalli S, et al. Transcriptomic profiling of the human brain reveals that altered synaptic gene expression is associated with chronological aging. Sci Rep. 2017;7:16890.

17. Rothman DL, Petroff OA, Behar KL, Mattson RH. Localized 1H NMR measurements of gamma-aminobutyric acid in human brain in vivo. Proc Natl Acad Sci USA. 1993;90:5662-6.

18. Gao F, Edden RA, Li M, Puts NA, Wang G, Liu C, et al. Edited magnetic resonance spectroscopy detects an age-related decline in brain GABA levels. Neuroimage. 2013;78:75-82.

19. Porges EC, Woods AJ, Edden RA, Puts NA, Harris AD, Chen $H$, et al. Frontal gamma-aminobutyric acid concentrations are associated with cognitive performance in older adults. Biol Psychiatry Cogn Neurosci Neuroimaging. 2017;2:38-44.

20. Rowland LM, Krause BW, Wijtenburg SA, McMahon RP, Chiappelli J, Nugent KL, et al. Medial frontal GABA is lower in older schizophrenia: a MEGA-PRESS with macromolecule suppression study. Mol Psychiatry. 2016;21:198-204.

21. Huang D, Liu D, Yin J, Qian T, Shrestha S, Ni H. Glutamate-glutamine and GABA in brain of normal aged and patients with cognitive impairment. Eur Radiol. 2017;27:2698-705.

22. Aufhaus E, Weber-Fahr W, Sack M, Tunc-Skarka N, Oberthuer G, Hoerst M, et al Absence of changes in GABA concentrations with age and gender in the human anterior cingulate cortex: a MEGA-PRESS study with symmetric editing pulse frequencies for macromolecule suppression. Magn Reson Med. 2013;69:317-20.

23. Mooney RA, Cirillo J, Byblow WD. GABA and primary motor cortex inhibition in young and older adults: a multimodal reliability study. J Neurophysiol. 2017;118:425-33.

24. Porges EC, Woods AJ, Lamb DG, Williamson JB, Cohen RA, Edden RAE, et al. Impact of tissue correction strategy on GABA-edited MRS findings. Neuroimage. 2017;162:249-56.

25. Pitchaimuthu K, Wu QZ, Carter O, Nguyen BN, Ahn S, Egan GF, et al. Occipital GABA levels in older adults and their relationship to visual perceptual suppression. Sci Rep. 2017;7:14231.

26. Marenco S, Geramita M, van der Veen JW, Barnett AS, Kolachana B, Shen J, et al Genetic association of ErbB4 and human cortical GABA levels in vivo. J Neurosci. 2011;31:11628-32.

27. Marenco S, Savostyanova AA, van der Veen JW, Geramita M, Stern A, Barnett AS, et al. Genetic modulation of GABA levels in the anterior cingulate cortex by GAD1 and COMT. Neuropsychopharmacology. 2010;35:1708-17.

28. Marenco S, Meyer C, Kuo S, van der Veen JW, Shen J, DeJong K, et al. Prefrontal GABA levels measured with magnetic resonance spectroscopy in patients with psychosis and unaffected siblings. Am J Psychiatry. 2016;173:527-34.

29. Dickinson D, Goldberg TE, Gold JM, Elvevag B, Weinberger DR. Cognitive factor structure and invariance in people with schizophrenia, their unaffected siblings, and controls. Schizophr Bull. 2011;37:1157-67.

30. Kaufman AS. Assessing adolescent and adult intelligence. Needham, MA: Allyn and Bacon; 1990

31. Geramita M, van der Veen JW, Barnett AS, Savostyanova AA, Shen J, Weinberger $\mathrm{DR}$, et al. Reproducibility of prefrontal gamma-aminobutyric acid measurements with J-edited spectroscopy. NMR Biomed. 2011;24:1089-98.

32. Shungu DC, Mao X, Gonzales R, Soones TN, Dyke JP, van der Veen JW, et al. Brain gamma-aminobutyric acid (GABA) detection in vivo with the J-editing (1) H MRS technique: a comprehensive methodological evaluation of sensitivity enhancement, macromolecule contamination and test-retest reliability. NMR Biomed. 2016;29:932-42.

33. Ashburner J, Friston K. Multimodal image coregistration and partitioning-a unified framework. Neuroimage. 1997;6:209-17.

34. Kochunov $\mathrm{P}$, Mangin JF, Coyle T, Lancaster J, Thompson P, Riviere D, et al. Agerelated morphology trends of cortical sulci. Hum Brain Mapp. 2005;26:210-20.

35. Baron RM, Kenny DA. The moderator-mediator variable distinction in social psychological research: conceptual, strategic, and statistical considerations. J Pers Soc Psychol. 1986;51:1173-82.

36. Hayes AF. Introduction to mediation, moderation, and conditional process analysis: a regression-based approach. New York, NY: Guilford Press; 2013. p. 507.

37. Nyhus E, Barcelo F. The Wisconsin Card Sorting Test and the cognitive assessment of prefrontal executive functions: a critical update. Brain Cogn 2009:71:437-51.

38. Salthouse TA, Fristoe N, Rhee SH. How localize are age-related effects on neuropsychological measures? Neuropsychology. 1996;10:272-85.

39. Axelrod BN, Jiron CC, Henry RR. Performance of adults ages 20 to 90 on the abbreviated Wisconsin card sorting test. Clin Neuropsychol. 1993;7:205-9.

40. Sedlacik J, Boelmans K, Lobel U, Holst B, Siemonsen S, Fiehler J. Reversible, irreversible and effective transverse relaxation rates in normal aging brain at $3 \mathrm{~T}$. Neuroimage. 2014;84:1032-41.

41. Tisell A, Leinhard OD, Warntjes JB, Lundberg P. Procedure for quantitative (1)H magnetic resonance spectroscopy and tissue characterization of human brain tissue based on the use of quantitative magnetic resonance imaging. Magn Reson Med. 2013;70:905-15.

42. Callaghan MF, Freund P, Draganski B, Anderson E, Cappelletti M, Chowdhury R, et al. Widespread age-related differences in the human brain microstructure revealed by quantitative magnetic resonance imaging. Neurobiol Aging 2014;35:1862-72.

43. Petroff OA, Errante LD, Rothman DL, Kim JH, Spencer DD. Neuronal and glial metabolite content of the epileptogenic human hippocampus. Ann Neurol. 2002;52:635-42.

44. Gomar JJ, Gordon ML, Dickinson D, Kingsley PB, Ulug AM, Keehlisen L, et al. APOE genotype modulates proton magnetic resonance spectroscopy metabolites in the aging brain. Biol Psychiatry. 2014;75:686-92.

45. Lehmann K, Steinecke A, Bolz J. GABA through the ages: regulation of cortical function and plasticity by inhibitory interneurons. Neural Plast. 2012;2012:892784.

46. ladarola MJ, Gale K. Cellular compartments of GABA in brain and their relationship to anticonvulsant activity. Mol Cell Biochem. 1981;39:305-29.

47. Wood JD, Kurylo E, Lane R. gamma-Aminobutyric acid release from synaptosomes prepared from rats treated with isonicotinic acid hydrazide and gabaculine. J Neurochem. 1988;50:1839-43.

48. Wu Y, Wang W, Richerson GB. GABA transaminase inhibition induces spontaneous and enhances depolarization-evoked GABA efflux via reversal of the GABA transporter. J Neurosci. 2001;21:2630-9.

49. Carpenter HE, Kelly KB, Bizon JL, Frazier CJ. Age-related changes in tonic activation of presynaptic versus extrasynaptic gamma-amniobutyric acid type $B$ receptors in rat medial prefrontal cortex. Neurobiol Aging. 2016;45:88-97.

50. Martinez-Losa M, Tracy TE, Ma K, Verret L, Clemente-Perez A, Khan AS, et al. Nav1.1-overexpressing interneuron transplants restore brain rhythms and cognition in a mouse model of Alzheimer's disease. Neuron. 2018;98:75-89.e5.

51. Verret L, Mann EO, Hang GB, Barth AM, Cobos I, Ho K, et al. Inhibitory interneuron deficit links altered network activity and cognitive dysfunction in Alzheimer model. Cell. 2012;149:708-21.

52. Kapogiannis D, Mattson MP. Disrupted energy metabolism and neuronal circuit dysfunction in cognitive impairment and Alzheimer's disease. Lancet Neurol. 2011;10:187-98.

53. Stranahan AM, Mattson MP. Bidirectional metabolic regulation of neurocognitive function. Neurobiol Learn Mem. 2011;96:507-16.

54. Kann O, Papageorgiou IE, Draguhn A. Highly energized inhibitory interneurons are a central element for information processing in cortical networks. J Cereb Blood Flow Metab. 2014;34:1270-82. 